Nervenarzt 2015 • 86:1007-1017 DOI 10.1007/s00115-015-4398-2

Online publiziert: 30 . Juli 2015

(c) The Author(s). This article is published with open access at link.springer.com
Bei der amyotrophen Lateralsklerose (ALS) hat die Hilfsmittelversorgung (HMV) eine zentrale Bedeutung. Seit 2011 wird die HMV von 4 ALS-Zentren in einem internetunterstützten Versorgungsnetzwerk koordiniert. Die Digitalisierung der HMV-Prozesse ermöglicht eine Auswertung der Versorgungsrealität im Vergleich unterschiedlicher ALS-Zentren, Hilfsmittelgruppen und Kostenträger. Die Analyse der HMV ist ein wesentlicher Schritt, um Behandlungsstandards der HMV zu definieren. Die Ergebnisse haben praktische Implikationen für die HMV bei der ALS und eine gesundheitspolitische Dimension.

\section{Hintergrund und Fragestellung}

Die HMV ist ein zentrales Element der symptomatischen und palliativen Be-

A. Funke' $\cdot$ T. Grehl ${ }^{2}$.J. Großkreutz ${ }^{3}$ C. Münch ${ }^{4,5} \cdot$ B. Walter ${ }^{4}$ D. Kettemann ${ }^{1}$.

C. Karnapp ${ }^{4} \cdot$ N. Gajewski ${ }^{5} \cdot$ R. Meyer ${ }^{1} \cdot$ A. Maier ${ }^{4} \cdot$ K.M. Gruhn ${ }^{2} \cdot$ T. Prell ${ }^{3}$.

K. Kollewe ${ }^{6} \cdot$ S. Abdulla ${ }^{6} \cdot$ X. Kobeleva ${ }^{6} \cdot$ S. Körner ${ }^{6} \cdot$ S. Petri ${ }^{6} \cdot$ T. Meyer $^{1,4,5}$

${ }^{1}$ Ambulanz für ALS und andere Motoneuronerkrankungen, Neurologische

Poliklinik, CharitéCentrum für Neurologie, Neurochirurgie und Psychiatrie, Charité

- Universitätsmedizin Berlin, Campus Virchow-Klinikum, Berlin

${ }^{2}$ Neurologische Klinik und Poliklinik, Ambulanz für ALS und andere Motoneuronerkrankungen, Berufsgenossenschaftliches Universitätsklinikum Bergmannsheil GmbH, Bochum

${ }^{3}$ Hans-Berger-Klinik für Neurologie, Ambulanz für neuromuskuläre und

Motoneuron-Erkrankungen/ALS, Universitätsklinikum Jena

${ }^{4}$ Neurologische Hochschulambulanz, Projektgruppe Ambulanzpartner,

Charité - Universitätsmedizin Berlin, Berlin

${ }^{5}$ Ambulanzpartner Soziotechnologie GmbH (APST), Berlin

${ }^{6}$ Neurologische Klinik mit Klinischer Neurophysiologie, Medizinische Hochschule Hannover

\title{
Hilfsmittelversorgung bei der amyotrophen Lateralsklerose
}

\section{Analyse aus 3 Jahren Fallmanagement in einem internetunterstützten Versorgungsnetzwerk}

handlung bei der ALS. Trotz ihrer sozialmedizinischen Relevanz wurde die HMV bei der ALS bisher nicht systematisch untersucht [1]. In der vorliegenden Studie wird die HMV an 4 ALS-Zentren in einem Zeitraum von 3,5 Jahren ausgewertet. Diese Daten erlauben erstmalig einen Direktvergleich („Benchmarking“) der Versorgungspraktiken unterschiedlicher ALS-Spezialambulanzen sowie der Kostenträger (Kranken- und Ersatzkassen sowie private Krankenversicherungen) im Hinblick auf Ablehnungsraten und Versorgungslatenzen bei definierten Hilfsmitteln (HM).

In der vorliegenden Untersuchung werden folgende Fragen für 4 ausgewählte ALS-typische HM-Gruppen bearbeitet:

- Wie ist die Versorgungsrate, d. h. der Anteil der tatsächlich gelieferten HM im Vergleich zu den ärztlich indizierten HM?
- Wie hoch ist die Ablehnungsrate der verschiedenen HM durch Patienten selbst und die Kostenträger?

- Wie lang ist die Versorgungslatenz von der ärztlichen Indikationsstellung bis zur Lieferung eines HM durch den Versorger?

\section{Methoden}

Die HMV an den ALS-Ambulanzen der Berufsgenossenschaftlichen Universitätsklinik Bergmannsheil (BGB) in Bochum, der Charité - Universitätsmedizin Berlin (Charité), der Medizinischen Hochschule Hannover (MHH) sowie des Universitätsklinikums Jena (UKJ) wurde für den Zeitraum von Juni 2011 bis Oktober 2014 ausgewertet ( Tab. 1). Die Datenerfassung

A. Funke, T. Grehl, J. Großkreutz haben gleichermaßen zu dem Artikel beigetragen. 
erfolgte auf Basis eines informierten Einverständnisses der Patienten.

Bei Ambulanzpartner (AP) handelt es sich um ein Konzept des ambulanten Versorgungsmanagements, das ein Fallmanagement mit einem internetbasierten Managementportal (http://www. ambulanzpartner.de) kombiniert. Der HM-Bedarf wurde im direkten Arzt-Patienten-Kontakt ermittelt. Er wurde auf einem Formblatt des AP-Netzwerkes markiert, das ein Auswahlmenü von ALS-typischen HM-Gruppen beinhaltet (Mobilitäts-, Transfer-, Pflege- und Kommunikationshilfen, Orthesen u. a.). In Freitextfeldern können zusätzliche HM und Konkretisierungen sowie Präferenzen der Patienten zu HM und Versorgern dokumentiert werden. Das Formular wird per Fax oder Scan an das "Datenmanagement“ versendet. Dabei handelt es sich um speziell geschulte Personen, die für die Digitalisierung des Formblattes verantwortlich sind.

Der entstandene Datensatz (sozialmedizinische Daten und HM-Bedarfe des Patienten) wird in einer digitalen Versorgungskette des Portals an einen Fallmanager $(\mathrm{w} / \mathrm{m})$ weitergeleitet. Dieser erfasst den Versorgungsbedarf und ordnet das $\mathrm{HM}$ einem geeigneten Versorger zu. Die Kriterien der Zuordnung sind die Wohnortnähe, Subspezialisierung und Vertragsverhältnisse des HM-Versorgers in Bezug auf den Versicherten und das HM. Dieses Fallmanagement erfolgt im Auftrag des Patienten. Der Patient hat jederzeit die Möglichkeit, von der Versorgung durch Netzwerkpartner zurückzutreten oder Versorger der eigenen Wahl zu beauftragen. Mit der Zuordnung eines HMBedarfs zu einem Leistungserbringer erhält der Versorger einen Zugang zu den versorgungsrelevanten Daten auf dem AP-Portal. Der Leistungserbringer kontaktiert den Patienten und vereinbart eine Beratung und Erprobung. Auf dieser Basis wird eine ärztliche Verordnung erstellt und an den Leistungserbringer versendet. Das Dokument wird beim Kostenträger zur Prüfung der medizinischen Indikation und Wirtschaftlichkeit eingereicht. Durch den Kostenträger erfolgt eine Genehmigung oder Ablehnung der HMV. Bei einer Genehmigung wird das HM beim Patienten ausgeliefert und in Be-

Nervenarzt 2015 • 86:1007-1017 DOI 10.1007/s00115-015-4398-2

(c) The Author(s). This article is published with open access at link.springer.com

A. Funke · T. Grehl · J. Großkreutz · C. Münch · B. Walter · D. Kettemann · C. Karnapp · N. Gajewski · R. Meyer · A. Maier · K.M. Gruhn · T. Prell · K. Kollewe · S. Abdulla · X. Kobeleva · S. Körner · S. Petri $\cdot$ T. Meyer Hilfsmittelversorgung bei der amyotrophen Lateralsklerose. Analyse aus 3 Jahren Fallmanagement in einem internetunterstützten Versorgungsnetzwerk

\section{Zusammenfassung}

Hintergrund. Die Hilfsmittelversorgung (HMV) ist ein zentrales Element in der Behandlung der amyotrophen Lateralsklerose (ALS). Seit 2011 wird die HMV an den universitären ALS-Zentren in Berlin, Bochum, Hannover und Jena in einem internetunterstützten Versorgungsnetzwerk koordiniert. Die Digitalisierung der HMV ermöglicht eine effiziente Auswertung der Versorgungsrealität. Zielstellung. Die Hilfsmittel (HM) in den Gruppen „Orthesen“ (OT), „, unterstützte Kommunikation" (UK), ,"therapeutische Bewegungsgeräte“ (TB) und „Elektrorollstühle" (ER) wurden hinsichtlich der Versorgungsrate (Anteil der ausgelieferten an den indizierten HM), der Ablehnung durch Patienten und Kostenträger sowie der Versorgungslatenz analysiert.

Ergebnisse. Im Zeitraum Juni 2011 bis Oktober 2014 wurden 1479 ALS-Patienten mit 12.478 HM koordiniert - davon $3313 \mathrm{HM}$ in den Gruppen OT, UK, TB und ER. Die mittlere Versorgungsrate dieser HM betrug 64,3\%. Eine Ablehnung der HMV durch Patienten selbst fand sich bei 9,8\% (OT 5,4\%; UK 9,8\%; TB 10,2\%; ER 15,6\%). In der Ablehnungsrate durch Kostenträger und der Versorgungslatenz ergaben sich erhebliche Unterschiede: OT (16,2\%; 68 Tage, $n=734)$, UK (30,4\%; 96 Tage, $n=392)$, TB (34,8\%; 113 Tage, $n=164)$ sowie $\operatorname{ER}(35,6 \% ; 129$ Tage, $n=259)$. Die Ablehnungsraten zeigten signifikante Unterschiede zwischen den Kostenträgern.

Schlussfolgerung. Insgesamt $64 \%$ der ärztlich indizierten HM erreichten die Patienten. Die Ablehnungsraten durch Patienten und Kostenträger sowie die Versorgungslatenzen waren hoch. Die Leistungsunterschiede zwischen Kostenträgern können erheblich sein. Eine wichtige Zukunftsaufgabe ist die Entwicklung von Indikationskriterien der HMV und ihre Integration in Behandlungspfade und Leitlinien der Fachgesellschaften.

\section{Schlüsselwörter}

Amyotrophe Lateralsklerose . Hilfsmittelversorgung $\cdot$ Kostenträger . Ablehnungsrate $\cdot$ Versorgungslatenz

\section{Provision of assistive devices in amyotrophic lateral sclerosis. Analysis of 3 years case management in an internet-based supply network}

\section{Summary}

Background. The provision of assistive devices (PAD) is a key element of care in amyotrophic lateral sclerosis (ALS). Since 2011, assistive devices (AD) have been coordinated in an internet-supported care network at university-based ALS centers in Berlin, Bochum, Hannover and Jena. The digitization of PAD processes has facilitated the evaluation of real-life ALS care.

Objectives. Orthotics (OT), augmentative and alternative communication (AAC), supported treadmill (ST) and powered wheelchair (PW) were the PAD groups analyzed for delivery rates (proportion of delivered $A D$ vs. medically indicated $A D$ ), rejection by patients and payers and latency of provision of care. Results. Between June 2011 and October 2014 a total of 1479 patients and 12,478 AD were coordinated, among which 3313 PAD were related to OT, AAC, ST or EM. The median delivery rate was $64.3 \%$. The mean rejection rate by patients was $9.8 \%$ (OT $5.4 \%$, AAC 9.8\%, ST 10.2\% and PW 15.6\%). Marked differences were noted in the rejection rate by payers and in care provision latency: OT (16.2\%, 68 days, $n=734)$, AAC $(30.4 \%, 96$ days, $\mathrm{n}=392)$, ST (34.8\%, 113 days, $\mathrm{n}=164)$ and PW (35.6\%, 129 days, $n=259)$. Analysis of rejection rates showed significant differences among insurers.

Conclusion. Only two thirds of the medically indicated AD reached the patients. Rejection rates by patients and payers and latency of provision of care were high. The PAD can substantially vary among health insurance companies. The establishment of consented criteria for PAD and their integration into treatment regimens and guidelines are crucial tasks for the future.

\section{Keywords}

Amyotrophic lateral sclerosis · Provision of assistive devices $\cdot$ Health insurance companies - Rejection rate - Latency of care provision 


\section{Hier steht eine Anzeige.}

\section{iㅡㄹ Springer}

trieb gesetzt. Die genannten Versorgungsschritte werden auf dem Portal digital dokumentiert und prozessiert ( $\bullet$ Tab. 2) sowie durch den Fallmanager inhaltlich und zeitlich koordiniert. Durch die Nutzung von AP werden die Daten und Prozesse in standardisierter Weise digitalisiert und den beteiligter Personenrollen ( $\mathrm{Pa}$ tient, Arzt, Koordinator, Leistungserbringer) bereitgestellt. Die Organisationsform, Datenschutzkonzeption, technische Umsetzung und Finanzierung von AP wurde an anderer Stelle beschrieben [2,3].

\section{Stichprobe}

Einschlusskriterien waren eine schriftliche Einwilligung des Patienten und die Diagnose einer ALS (ICD-10: G12.2) einschließlich der klinischen Varianten [4, 5, 6]. Erfasst wurden sämtliche HM, die innerhalb des AP-Netzwerkes versorgt wurden. Die Analyse der HMV wurde dabei auf 4 ausgewählte HM-Gruppen beschränkt: Orthesen (die Gesamtheit von Arm-, Bein- und Zervikalorthesen), therapeutische Bewegungsgeräte („Bewegungstrainer" der unteren und oberen Extremitäten mit Elektroantrieb zur passiven Bewegung paretischer Extremitäten), HM der unterstützten Kommunikation (einschließlich „Tablet-Computern“ sowie hand-, kopf- und augengesteuerten Kommunikationshilfen) und Elektrorollstühle (einschließlich Leichtgewicht- und Multifunktionsrollstühlen mit elektrischer Schiebehilfe). Die Auswahl der analysierten HM-Gruppen orientierte sich an ihrer Relevanz, Häufigkeit und Kostenintensität $[7,8,9,10,11]$. Orthesen und therapeutische Bewegungsgeräte werden mit einer supportiven Zielstellung zum Erhalt oder zur Stärkung motorischer Funktionen eingesetzt. Die HMV mit unterstützter Kommunikation und Elektrorollstühlen dient der Kompensation motorischer Defizite in den Dimensionen der Kommunikation und Mobilität. Mit dieser HM-Auswahl wurden unterschiedliche Dimensionen der ALS-HMV abgebildet.

\section{Untersuchungsmethoden und Analysekriterien}

Eine Übersicht der analysierten Daten im Prozess der HMV findet sich in $\bullet$ Tab. 2. 
Tab. 1 Hilfsmittelversorgung an 4 ALS-Zentren im Vergleich

\begin{tabular}{|c|c|c|c|c|c|}
\hline Zentrum & BGB & Charité & МHH & UKJ & Gesamt \\
\hline Projektbeginn & Juni 2011 & August 2011 & August 2013 & Juni 2012 & \\
\hline Patientenzahl & 232 & 941 & 65 & 164 & 1479 \\
\hline \multicolumn{6}{|l|}{ Orthetik } \\
\hline Indiziert (n) & 149 & 952 & 28 & 135 & 1264 \\
\hline Versorgt $(n)$ & 126 & 741 & 13 & 88 & 968 \\
\hline Versorgungsrate (\%) & 84,6 & 77,8 & 46,4 & 65,2 & 76,6 \\
\hline \multicolumn{6}{|c|}{ Unterstützte Kommunikation } \\
\hline Indiziert (n) & 113 & 654 & 35 & 110 & 912 \\
\hline Versorgt $(n)$ & 69 & 395 & 20 & 63 & 547 \\
\hline Versorgungsrate (\%) & 61,1 & 60,4 & 57,1 & 57,3 & 60,0 \\
\hline \multicolumn{6}{|c|}{ Therapeutische Bewegungsgeräte } \\
\hline Indiziert (n) & 30 & 300 & 8 & 65 & 403 \\
\hline Versorgt (n) & 21 & 168 & 3 & 39 & 231 \\
\hline Versorgungsrate (\%) & 70,0 & 56,0 & 37,5 & 60,0 & 57,3 \\
\hline \multicolumn{6}{|l|}{ Elektrorollstühle } \\
\hline Indiziert (n) & 82 & 566 & 29 & 57 & 734 \\
\hline Versorgt (n) & 48 & 291 & 15 & 29 & 383 \\
\hline Versorgungsrate (\%) & 58,5 & 51,4 & 51,7 & 50,9 & 52,2 \\
\hline \multicolumn{6}{|c|}{ Hilfsmittelgruppen (gesamt) } \\
\hline Indiziert (n) & 374 & 2472 & 100 & 367 & 3313 \\
\hline Versorgt (n) & 264 & 1595 & 51 & 219 & 2129 \\
\hline Versorgungsrate (\%) & 70,6 & 64,5 & 59,7 & 51,0 & 64,3 \\
\hline
\end{tabular}

Tab. 2 Datensätze zur Analyse der Hilfsmittelversorgung

\begin{tabular}{|ll}
\hline Datensatz & Beschreibung \\
\hline Diagnose & Nach ICD-10 \\
\hline Krankenversicherung & Kostenträger der Gesetzlichen oder Privaten Krankenversicherung \\
\hline Wohnort & Postleitzahl; für Zuordnung zum ALS-Zentrum \\
\hline Hilfsmittelgruppe & $\begin{array}{l}\text { Kategorisierung der Hilfsmittel (hier in Orthesen, unterstützte } \\
\text { Kommunikation, therapeutische Bewegungsgeräte und Elektro- } \\
\text { rollstühle) }\end{array}$ \\
\hline Spezifikation des Hilfsmittels & $\begin{array}{l}\text { Detaillierte Beschreibung gemäß Hilfsmittelkatalog des GKV- } \\
\text { Spitzenverbandes }\end{array}$ \\
\hline Name des Hilfsmittels & Produktbezeichnung mit Handelsnamen \\
\hline Versorgungsanfrage & Datum der Veranlassung der Hilfsmittelversorgung durch den Arzt \\
\hline Lieferung & $\begin{array}{l}\text { Datum der Auslieferung des Hilfsmittels und Herstellen der } \\
\text { Gebrauchsfähigkeit }\end{array}$ \\
\hline Versorgungslatenz & $\begin{array}{l}\text { Wird aus dem Zeitintervall (in Tagen) zwischen Versorgungsanfrage } \\
\text { und Lieferung des Hilfsmittels ermittelt }\end{array}$ \\
\hline Ablehnung der Versorgung & Datum einer Ablehnung der Hilfsmittelversorgung \\
\hline Ablehnungsgründe & $\begin{array}{l}\text { Auswahlmenü standardisierter Ablehnungsgründe durch } \\
\text { Patienten und Kostenträger }\end{array}$ \\
\hline ALS amyotrophe Lateralsklerose, GKV Gesetzliche Krankenversicherung. \\
\hline
\end{tabular}

Aus der Zeitdifferenz zwischen der Indikationsstellung der HMV und dem Lieferdatum wurde die Versorgungslatenz in Tagen ermittelt. Der Anteil der gelieferten HM zur Gesamtheit der ärztlich indizierten HM wurde als Versorgungsrate defi- niert. Die Ablehnung einer HMV wurde in einem gesonderten Statusschritt dokumentiert (Datum; Ablehnung durch Patient oder Kostenträger; Gründe der Ablehnung). Berücksichtigt wurden ausschließlich endgültige Ablehnungen. In- itiale Ablehnungen, die durch Widerspruchsverfahren revidiert wurden, sind nicht als Ablehnung gewertet worden. Der Anteil der abgelehnten HM im Vergleich zur Gesamtheit der ärztlich indizierten HM wurde als Ablehnungsrate bezeichnet. In der Analyse der Krankenkassen wurden die fünf häufigsten Kostenträger der Gesetzlichen Krankenversicherung (GKV) berücksichtigt: AOK - Die Gesundheitskasse (AOK); Techniker Krankenkasse (TK); BARMER GEK (BEK) und DAK - Gesundheit (DAK). Aufgrund der geringen Patientenzahlen der einzelnen Betriebs- (BKK) und Innungskrankenkassen (IKK) wurden diese Versicherten in der Gruppe der BKK (mit IKK) zusammengefasst. Analog wurde mit Versicherten der Privaten Krankenversicherungen (PKV) verfahren (Gruppe der PKV).

\section{Datenanalyse}

Für die Analyse der indizierten HM wurde eine Grundgesamtheit von 3313 HMV untersucht. Bei der Ermittlung der Ablehnungsraten (durch Patienten und Kostenträger) wurden alle $\mathrm{HMV}$, bei denen andere Gründe zu einer Nichtversorgung führten (Patient verstorben; HM medizinisch ungeeignet etc.) aus der Stichprobe ausgeschlossen (resultierende Stichprobe: $3192 \mathrm{HM}$ ). Bei den Ablehnungsraten durch die Kostenträger wurden alle HMV, die vom Patienten abgelehnt wurden, nicht in der Stichprobe berücksichtigt (resultierende Stichprobe: 2880 HM). In der Berechnung der Versorgungslatenz wurden gelieferte HMV ausgeschlossen, deren Lieferdatum nicht dokumentiert war (resultierende Stichprobe: 1545 HM).

Die statistische Untersuchung erfolgte mit IBM SPSS Statistics 22.0 (IBM Corporation, Armonk, USA). Für die Berechnungen der Latenzen wurden Mediane sowie Mittelwerte mit Standardfehlern des Mittelwerts ermittelt. Unterschiede zwischen den Versorgungslatenzen wurden mithilfe des Kruskal-Wallis-Tests untersucht. Für den Vergleich der Ablehnungsraten wurden die Kostenträger paarweise mit dem $\chi^{2}$-Test nach Pearson verglichen. 


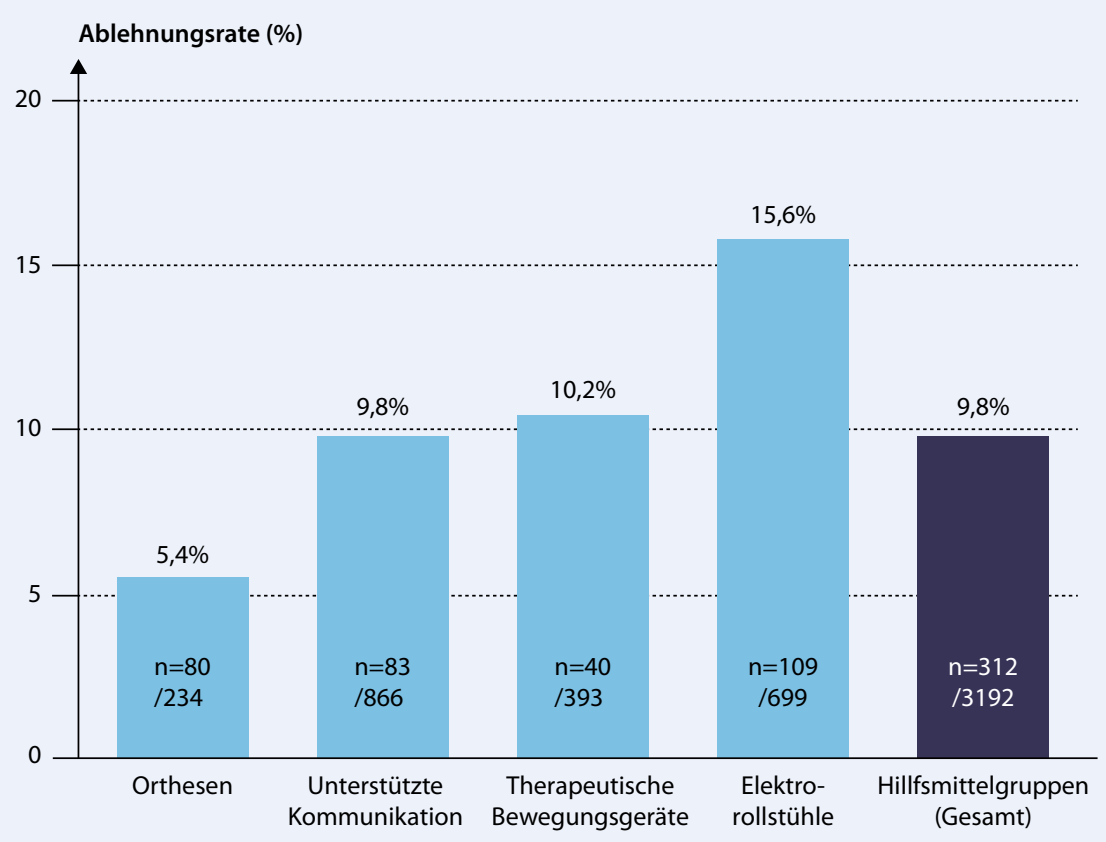

Abb. $1 \Delta$ Ablehnung der Hilfsmittelversorgung durch Patienten im Vergleich der Hilfsmittelgruppen

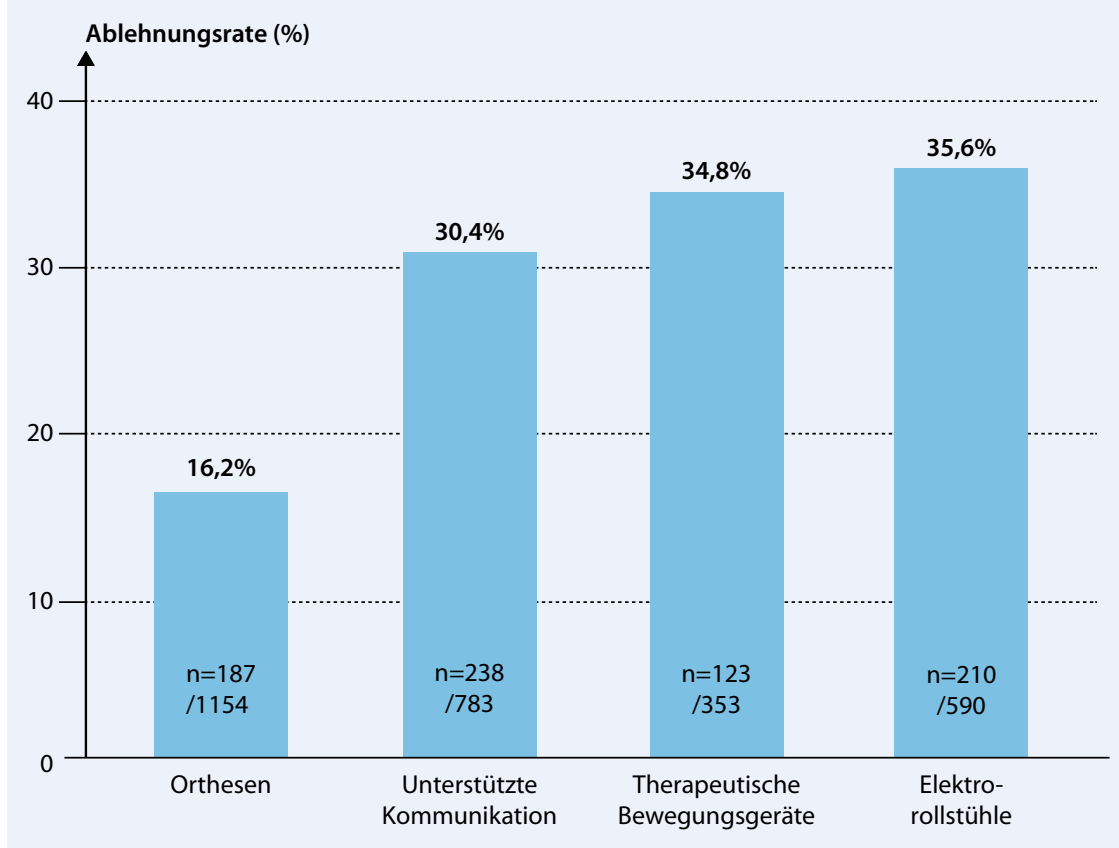

Abb. $2 \Delta$ Ablehnung der Hilfsmittelversorgung durch die Kostenträger im Vergleich der Hilfsmittelgruppen

\section{Ergebnisse}

\section{Charakteristika der Stichprobe}

Im AP-Netzwerk wurden im Untersuchungszeitraum 1479 ALS-Patienten versorgt. Dabei wurden insgesamt 12.478 HM koordiniert und systematisch erfasst. Die werks koordiniert. Dabei ergab sich die folgende Häufigkeitsverteilung (prozentualer Anteil an der Gesamtheit der analysierten HM): Charité $=2472 \mathrm{HM}(74,6 \%)$; BGB =374 HM (11,3\%); $\mathrm{MHH}=100 \mathrm{HM}$ (3\%); UKJ =367 HM (11,1\%; • Tab. 1). In den 5 häufigsten GKV und der Gesamtheit der PKV-Versicherten zeigte sich folgende Häufigkeitsverteilung der indizierten HM (prozentualer Anteil an der Gesamtheit der analysierten HM): $\mathrm{BKK}=792 \mathrm{HM}(23,9 \%)$; $\mathrm{AOK}=687 \mathrm{HM}$ $(20,7 \%) ; \mathrm{TK}=594 \mathrm{HM}(17,9 \%) ; \mathrm{BEK}$ $=574 \mathrm{HM}(17,3 \%) ; \mathrm{PKV}=364 \mathrm{HM}(11,0 \%)$ und DAK =302 HM (9,1\%).

\section{Versorgungsrate}

Die Versorgungsrate der untersuchten HM-Gruppen betrug für alle Zentren 64,3\%. Die Differenzierung der ALSZentren zeigte deutliche Unterschiede zwischen den Versorgungsraten: BGB $=70,6 \%$; Charité $=64,5 \%$; $\mathrm{MHH}=51,0 \%$ und UKJ $=59,7 \%$. Zwischen den HMGruppen ergaben sich erhebliche Unterschiede der mittleren Versorgungsrate: Orthesen $76,6 \%$, unterstützte Kommunikation $60,0 \%$, therapeutische Bewegungsgeräte 57,3\% und Elektrorollstühle 52,2\% (• Tab. 1).

\section{Ablehnung der HMV durch Patienten}

In - Abb. 1 ist die Ablehnungsrate der HMV durch Patienten dargestellt (insgesamt 9,8\%). Zwischen den HM-Gruppen ergaben sich deutliche Unterschiede der Ablehnungsrate. Die Versorgung mit Orthesen hatte eine sehr hohe Akzeptanz (Ablehnungsrate 5,4\%). Die HMV mit Elektrorollstühlen war mit einer hohen Ablehnungsrate durch Patienten verbunden (15,6\%). Die HMV mit unterstützter Kommunikation $(9,8 \%)$ und therapeutischen Bewegungsgeräten (10,2\%) zeigte intermediäre Werte. Die patientenseitige Ablehnung einer HMV betrug bei Versicherten der GKV 8,9-9,9\%. Das Ablehnungsverhalten durch Patienten war innerhalb der GKV damit recht einheitlich. Auffällig war jedoch eine deutlich höhere Ablehnung der HMV bei Versicherten der PKV (16,1\%). 
Die Ablehnungsrate der HMV durch die Kostenträger zeigt $\bullet$ Abb. 2. Dabei wurden die HMV-Prozesse ausgewertet, bei denen eine zustimmende oder ablehnende Entscheidung der Kostenträger dokumentiert und nachvollziehbar war. Von $2880 \mathrm{HM}$ wurden 758 von den Kostenträgern abgelehnt (26,3\%). Zwischen den Kostenträgern ergaben sich deutliche Unterschiede der Ablehnungsraten. Die TK $(18,9 \%)$ und DAK (20,8\%) zeigten im Vergleich die geringsten Ablehnungsraten, während die PKV $(34,8 \%)$ und BKK (33,0\%) die höchsten Ablehnungsraten aufwiesen.

\section{Vergleich der HM-Gruppen}

Zwischen den HM-Gruppen ergaben sich erhebliche Unterschiede der Ablehnungsrate durch die Kostenträger: Orthesen $16,2 \%$, unterstützte Kommunikation 30,4\%, therapeutische Bewegungsgeräte $34,8 \%$ und Elektrorollstühle 35,6\% (- Abb. 2). Für Orthesen zeigte sich damit eine relativ geringe Ablehnung durch Krankenkassen, während die anderen HM-Gruppen hohe Ablehnungsraten aufwiesen.

\section{Vergleich der Kostenträger}

Das Bewilligungs- und Ablehnungsverhalten der Kostenträger war different und heterogen (• Tab. 3 und $\bullet$ Abb. 3). So zeigte die AOK eine 2,5-fach erhöhte Ablehnungsrate der Orthesen $(25,4 \%)$ im Vergleich zur DAK (10,6\%). Im Gegensatz dazu fand sich für die unterstützte Kommunikation bei der AOK die geringste Ablehnungsrate (19,2\%), während die DAK einen hohen Ablehnungswert zeigte $(32 \%)$. Bei den therapeutischen Bewegungsgeräten ergaben sich insgesamt hohe Ablehnungsraten. Dennoch waren auch hier 2,3-fache Unterschiede zwischen den Kostenträgern nachweisbar (BKK 52,2\% vs. TK 22,1\%). Bei Elektrorollstühlen bestand die höchste Ablehnungsrate. Die Unterschiede zwischen den Kostenträgern waren erheblich (BKK $50,3 \%$ vs. TK $18,3 \%$ ). 
Tab. 3 Hilfsmittelversorgung im Vergleich der Krankenkassen - die Ablehnungsraten

\begin{tabular}{|c|c|c|c|c|c|c|c|}
\hline Kostenträger & AOK & BEK & BKK & DAK & PKV & TK & Gesamt \\
\hline \multicolumn{8}{|l|}{ Orthetik } \\
\hline Indiziert (n) & 228 & 205 & 274 & 113 & 118 & 216 & 1154 \\
\hline Abgelehnt (n) & 58 & 22 & 50 & 12 & 21 & 24 & 187 \\
\hline Ablehnungsrate (\%) & 25,4 & 10,7 & 18,2 & 10,6 & 17,8 & 11,1 & 16,2 \\
\hline \multicolumn{8}{|c|}{ Unterstützte Kommunikation } \\
\hline Indiziert (n) & 172 & 134 & 187 & 76 & 83 & 131 & 783 \\
\hline Abgelehnt (n) & 33 & 41 & 59 & 26 & 39 & 40 & 238 \\
\hline Ablehnungsrate (\%) & 19,2 & 30,7 & 31,6 & 34,2 & 47,0 & 30,5 & 30,4 \\
\hline \multicolumn{8}{|c|}{ Therapeutisches Bewegungsgeräte } \\
\hline Indiziert (n) & 74 & 62 & 92 & 25 & 32 & 68 & 353 \\
\hline Abgelehnt (n) & 18 & 21 & 48 & 8 & 13 & 15 & 123 \\
\hline Ablehnungsrate (\%) & 24,3 & 33,9 & 52,2 & 32,0 & 40,6 & 22,1 & 34,8 \\
\hline \multicolumn{8}{|l|}{ Elektrorollstühle } \\
\hline Indiziert (n) & 118 & 104 & 145 & 60 & 54 & 109 & 590 \\
\hline Abgelehnt (n) & 49 & 39 & 73 & 11 & 27 & 20 & 210 \\
\hline Ablehnungsrate (\%) & 41,5 & 28,8 & 50,3 & 18,3 & 50,0 & 18,3 & 35,6 \\
\hline \multicolumn{8}{|c|}{ Hilfsmittelgruppen (gesamt) } \\
\hline Indiziert (n) & 592 & 505 & 698 & 274 & 287 & 524 & 2880 \\
\hline Abgelehnt (n) & 158 & 114 & 230 & 57 & 100 & 99 & 758 \\
\hline Ablehnungsrate (\%) & 26,7 & 22,6 & 33,0 & 20,8 & 34,8 & 18,9 & 26,3 \\
\hline
\end{tabular}

\section{Versorgungslatenz}

Bei 468 HMV lagen inkomplette Datensätze vor (Lieferung erfolgt, aber Lieferdatum des HM nicht dokumentiert). Diese Datensätze wurden bei der Kalkulation der Versorgungslatenz nicht berücksichtigt. Die Analyse der Versorgungslatenz war auf die gelieferten HM mit kompletten Datensätzen beschränkt ( $\mathrm{n}=1545)$. Zwischen den HM-Gruppen ergaben sich erhebliche Unterschiede der mittleren Versorgungslatenz (• Abb.4): Orthesen $67,9 \pm 71,5$ Tage $(\mathrm{n}=734)$, unterstützte Kommunikation 96,1 $\pm 60,6$ Tage $(\mathrm{n}=392)$, therapeutische Bewegungsgeräte $113,3 \pm 70,9$ Tage $(\mathrm{n}=164)$ und Elektrorollstühle $129,7 \pm 84,6$ Tage $(\mathrm{n}=259)$. Zwischen einzelnen Kostenträgern fanden sich bei der unterstützen Kommunikation und Orthetik signifikante Unterschiede in der Versorgungslatenz (Kruskal-Wallis-Test; Signifikanzniveau $\mathrm{p}<0,05)$. Bei Elektrorollstühlen und therapeutischen Bewegungsgeräten bestanden keine signifikante Unterschiede (『 Abb.4).

\section{Diskussion}

Die vorliegende Untersuchung ist die erste systematische Analyse der HMV bei der ALS in Deutschland. Dabei wurden Daten aus 4 ALS-Zentren erfasst, die in einem digital unterstützten Versorgungsnetzwerk zusammenarbeiten. Eine Stärke der Untersuchung liegt in der Erfassung der Versorgungsrealität dieser Zentren. Das Fall-, Dokumenten- und Datenmanagement war standardisiert und digitalisiert. Die Verordnerstruktur war homogen (Fachärzte für Neurologie in spezialisierten ALS-Zentren). Diese Homogenität war zugleich eine Limitation der Untersuchung, da sie mit einer Selektion besonderer Krankheitsverläufe verbunden sein kann. Im Fokus der Analyse standen ausgewählte HM, um eine hohe Vergleichbarkeit zwischen ALS-Zentren und Kostenträgern zu erreichen. Zugleich blieben zahlreiche Fragestellungen noch unbearbeitet (z. B. die Häufigkeitsverteilung aller HM und ihre Differenzierung). Bei $22 \%$ der HMV (468 HM) konnte die Versorgungslatenz nicht analysiert werden, da die Lieferdaten nicht dokumentiert waren. Dabei ist zu spekulieren, dass insbesondere bei HM mit hoher Versorgungs- latenz die Lieferdaten nicht erfasst wurden und möglicherweise höhere Versorgungslatenzen vorlagen. Weitgehend unbekannt sind die Häufigkeiten und Ergebnisse sozialrechtlicher Widerspruchs- und Klageverfahren bei einer Ablehnung der HMV. In zukünftigen Studien wird von Interesse sein, ob die Ablehnungsraten und Latenzen der HMV einen Einfluss auf die Lebensqualität und das Überleben bei der ALS haben. Insbesondere die Versorgungsrate von Hilfsmitteln der Beatmungsversorgung ist ein potenzieller, aber noch nicht untersuchter Prognosefaktor bei der ALS.

Die Studie erlaubt erstmalig eine integrierte Betrachtung aller beteiligten Personenrollen der HMV (Ärzte, Patienten, Versorger, Kostenträger). Die HM-Analyse einzelner Kostenträger, z. B. des „Hilfsmittelreports" der BEK, beruht überwiegend auf Kostendaten [11, 12]. Patientenund arztseitige Faktoren sowie Daten zu Prozessen und Zeitabläufen sind den Krankenkassen nur eingeschränkt zugänglich. Diese Limitation wurde in der vorliegenden Studie überwunden. Die kostenträgerübergreifende Datenerfassung ermöglichte eine vergleichende Analyse unterschiedlicher Kranken- und Ersatzkassen.

Die Versorgungsrate in den ALS-Zentren variierte und reichte von $51,0-70,6 \%$ (• Tab. 1). Ursächlich kommen methodische Effekte infrage, da das AP-Versorgungsnetzwerk an den ALS-Ambulanzen zu unterschiedlichen Zeitpunkten (zwischen Juni 2011 und August 2013) initiiert wurde. Weiterhin bestanden an den Zentren unterschiedliche Versorgungsschwerpunkte. Dieser Faktor ist relevant, da die HMV eine ausreichende Ressourcenallokation erfordert (Beratung zu HM; Dokumentation, Begründung und Begutachtung von HMV). Neben der arztseitigen Indikationsstellung wird die Versorgungsrate durch Patienten, Leistungserbringer und Kostenträger bestimmt.

Die Ergebnisse zeigen eine Ablehnungsrate durch Patienten (9,8\%), die sich maßgeblich in den einzelnen HMGruppen unterscheidet. So ist die Akzep$\tan z$ von Orthesen sehr hoch (Ablehnung 5,4\%), während die Rollstuhlversorgung deutlich häufiger von Patienten abgelehnt wird (15,6\%; • Abb. 1). Die Ursachen der 

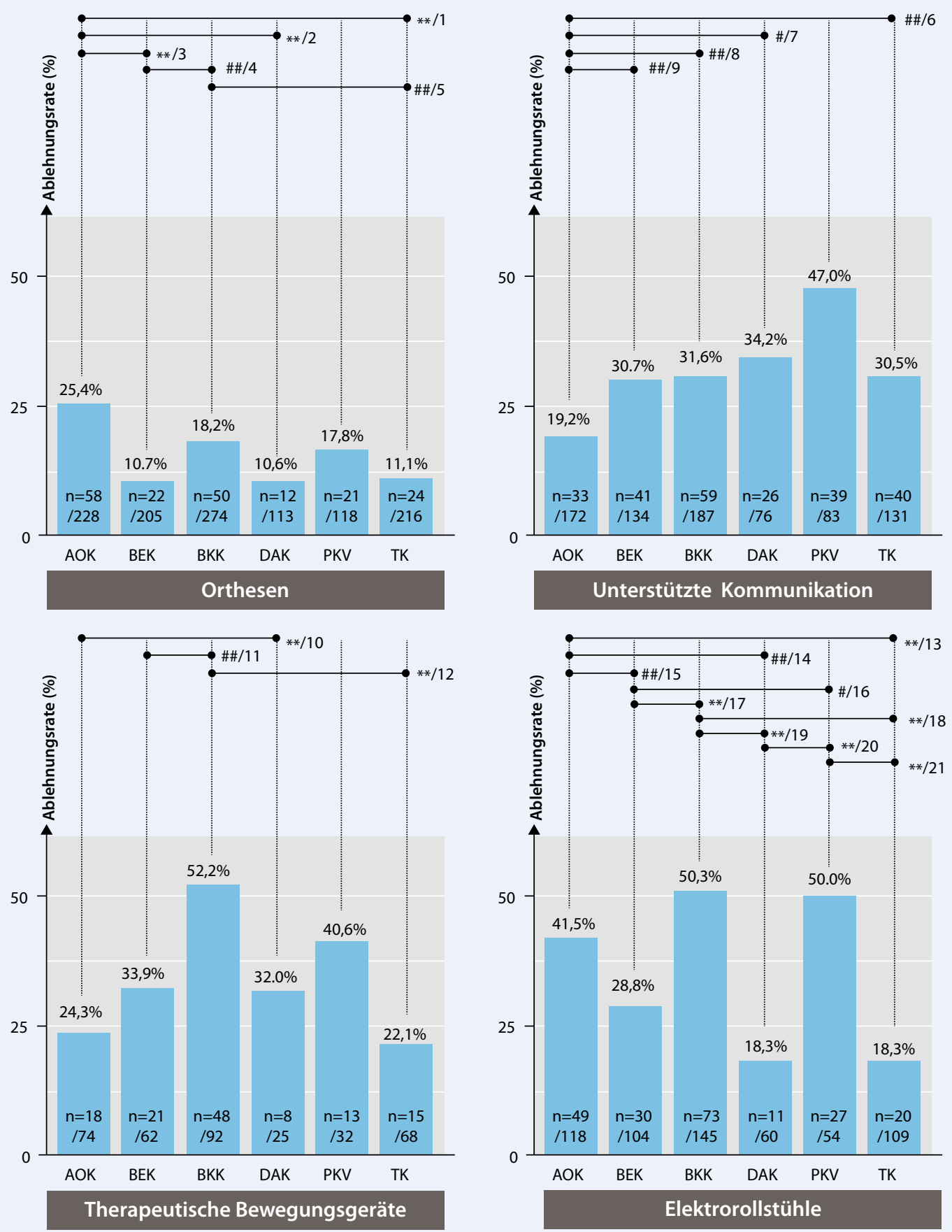

Abb. $3 \Delta$ Ablehnung der Hilfsmittelversorgung durch Krankenkassen im Vergleich der Kostenträger und Hilfsmittelgruppen. AOK AOK - Die Gesundheitskasse, BEK BARMER GEK, BKK Betriebskrankenkassen, DAK DAK - Gesundheit, PKV Private Krankenversicherung, TKTechniker Krankenkasse. Die Signifikanzniveaus für die Ablehnungsunterschiede zwischen Kostenträgern wurden mit einem Balken und den Symbole gekennzeichnet: **: $p<0,001 ; \#: p<0,01 ; \# \#: p<0,05$. Die Effektstärke wurde mit der Odds Ratio (OR) und den 95\%-Konfidenzintervall erfasst und mit laufenden Ziffern (1-21) dem betrachteten statistischen Zusammenhang (Balken) zugeordnet. Für Orthesen: 1: AOK/TK 2,729 (1,625-4,584); 2: AOK/DAK 2,872 (1,472-5,604); 3: AOK/BEK 2,838 (1,665-4,838); 4: BKK/BEK 1,855 (1,08-3,185); 5: BKK/TK 1,786 (1,058-3,014); für unterstützte Kommunikation: 6: TK/AOK 1,852 (1,088-3,155); 7: DAK/AOK 2,188 (1,193-4,016); 8: BKK/AOK 1,941 (1,19-3,165); 9: AOK/BEK 1,855 (1,095-3,145); für therapeutische Bewegungsgeräte: 10: AOK/DAK 3,39 (1,736-6,623); 11: BKK/BEK 2,128 (1,094-4,149); 12 : BKK/TK 3,855 (1,906-7,795); für Elektrorollstühle: 13: AOK/TK 3,45 (1,803-6,601); 14: AOK/DAK 2,946 (1,321-6,573); 15: AOK/ BEK 2, 166 (1,175-3,993); 16: PKV/BEK 2,469 (1,248-4,878); 17: BKK/BEK 2,469 $(1,445-4,219)$; 18: BKK/TK 4,45 $(2,48-7,986)$; 19: BKK/DAK 4,455 (2,145-9,253); 20: PKV/DAK 4,454 (1,916-10,309); 21: PKV/TK 4,45 (2,164-9,151) 

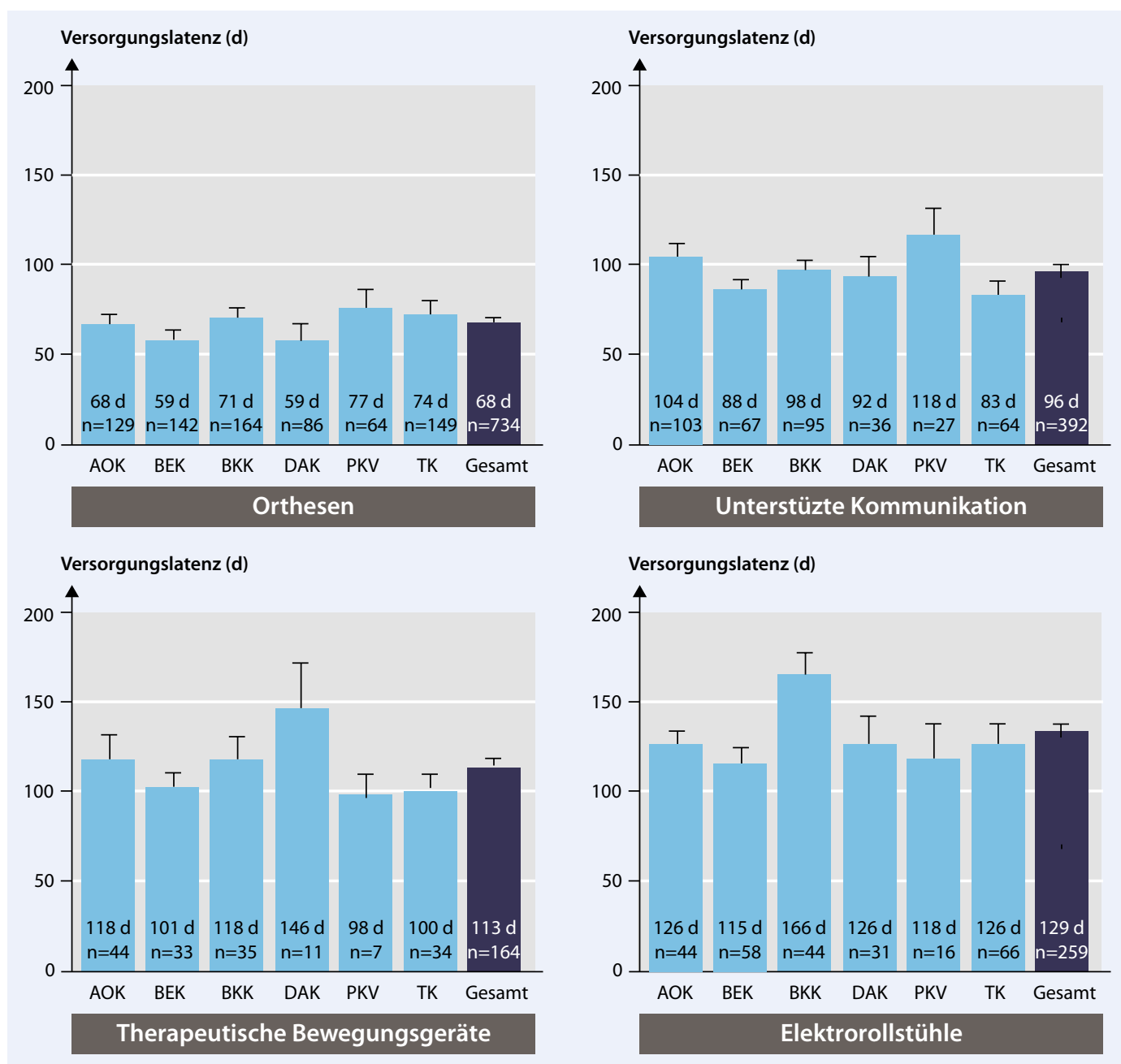

\begin{abstract}
Abb. $4<$ Versorgungslatenz im Vergleich der Hilfsmittelgruppen und Kostenträger (Mittelwerte). AOK AOK - Die Gesundheitskasse, BEK BARMER GEK, BKK Betriebskrankenkassen, DAK DAK - Gesundheit, PKV Private Krankenversicherung, TKTechniker Krankenkasse
\end{abstract}

patientenseitigen Ablehnung wurden bisher nicht im Detail analysiert. Zu diskutieren ist eine subjektiv erlebte Stigmatisierung durch HM (z. B. Rollstuhlnutzung), die fehlende Infrastruktur der HMV (z. B. fehlende Unterstellmöglichkeit für Elektrorollstühle in städtischen Wohnlagen) oder das subjektive Erleben von HM als „Meilenstein“ einer progredienten Erkrankung. Ein weiterer Faktor der Unterversorgung kann die Frustration von Patienten durch die Verzögerung der HMV sein [13], die in der Datenbanksystematik dieser Studie als „Ablehnung durch Patienten“ klassifiziert wurde. In diesem Zusammenhang ist auffällig, dass die PKV die höchste patientenseitige $(16,1 \%)$ Ablehnung der HMV zeigte. Dabei sind sozioökonomische Faktoren (z. B. Belastungen durch das Kostenerstattungsprinzip) zu diskutieren. Die Ermittlung der patientenseitigen Ablehnungsgründe, das reale Nutzungsverhal- ten und die Zufriedenheit mit Versorgungsoptionen sind Gegenstand weiterer Untersuchungen.

In den analysierten HM-Gruppen ergaben sich deutliche Unterschiede in der Ablehnungsrate durch Kostenträger und der Versorgungslatenz. Bei Orthesen fand sich die geringste Ablehnungsrate (16,2\%) und Versorgungslatenz (68 Tage). Die HM-Gruppen der unterstützten Kommunikation und therapeutischen Bewegungsgeräte ergaben etwa doppelt so hohe Ablehnungsraten (34,8 und 30,4\%) und deutlich höhere Versorgungslatenzen (113 und 96 Tage). Die für die ALS besonders relevante HMV mit Elektrorollstühlen zeigte die größte Problematik (Ablehnungsrate: 35,6\%; Versorgungslatenz: 129 Tage; Abb. 3; 4).

In der Ablehnungsrate der Kostenträger konnten erhebliche Unterschiede zwischen den einzelnen Kranken- und Ersatzkassen nachgewiesen werden. Insge- samt bestanden bei der TK und DAK die geringsten Ablehnungsraten. Vor allem in der HMV mit Elektrorollstühlen fand sich eine signifikant geringere Ablehnung bei der TK und DAK (beide 18,3\%) im Vergleich zur AOK (41,5\%), BKK (50,3\%) und PKV (50,0\%).

Die Daten zu Ablehnungsraten und zur Versorgungslatenz einzelner HM haben unmittelbare praktische Implikationen in der Betreuung von ALS-Patienten. Bereits in der Beratung zu den indizierten HM sollten die Patienten über die zu erwartende Ablehnungswahrscheinlichkeit und die Möglichkeit eines Widerspruchs im Falle einer Ablehnung informiert werden. Vor dem Hintergrund des progredienten Charakters der ALS ist das Konzept der „Vorabberatung“ zu HM mit hoher Versorgungslatenz (z. B. bei Kommunikationshilfsmitteln oder Elektrorollstühlen) anzubieten. 
Die Daten zur PKV entstanden durch die gemeinsame Betrachtung und Auswertung aller Kostenträger der PKV. Eine Differenzierung unterschiedlicher privater Krankenversicherungen war methodisch noch nicht möglich, da die Anzahl der Versicherten pro PKV zu gering war. Bei der GKV war die Analyse auf die häufigsten Kostenträger begrenzt. Eine Regionalisierung der Leistungsdaten für die GKV und PKV war ebenfalls noch nicht möglich. Entgegen der medialen Diskussion ergaben sich in unserer Untersuchung keine Hinweise auf eine „ZweiKlassen-Medizin“ zugunsten der PKV. Vielmehr zeigte die PKV in den untersuchten ALS-HM die höchste Ablehnungsrate. Allerdings fanden sich auch innerhalb der GKV signifikante Leistungsunterschiede, die sich mit divergierenden Ablehnungsraten (bis zu einem Faktor von 2,5) darstellten. Aufgrund der erheblichen Leistungsunterschiede müssen „Klassenunterschiede“ innerhalb der GKV diskutiert werden. Eine Reproduktion und öffentliche Bereitstellung dieser Daten hat das Potenzial für einen breiteren gesundheitspolitischen Diskurs.

Die hochsignifikanten Unterschiede in der Ablehnung von HM zwischen einzelnen GKV deuten auf Differenzen in Bewilligungsprozessen innerhalb der Krankenkassen hin. Die kasseninternen Entscheidungsprozesse der HMV sind weitgehend unbekannt. Die bestehende Diskrepanz in der arzt- und kostenträgerseitigen Einschätzung der HMV-Indikation ist ineffektiv und mit einem hohen Ressourcenverbrauch der beteiligten Personenrollen verbunden (Arzt, Patient, Leistungserbringer und Kostenträger; $[14,15])$. Die hohe systematische Ablehnungsrate und Versorgungslatenz in der HMV zeigt die Notwendigkeit einer Konsensbildung zwischen Fachgesellschaften und Kostenträgern [16]. Eine wichtige und ambitionierte Zukunftsaufgabe liegt in der Aufnahme der HMV in Behandlungspfade und neurologische Leitlinien, in denen Versorgungsziele, Indikationskriterien und Qualitätsmerkmale der HMV definiert werden.

\section{Fazit für die Praxis}

- Bei der ALS erreichen lediglich zwei Drittel der ärztlich indizierten Hilfsmittel die Patienten. Dabei bestehen erhebliche Unterschiede in der Versorgung mit Orthesen, Hilfsmitteln der unterstützten Kommunikation und Elektrorollstühlen. Wichtige Faktoren der Nichtversorgung liegen sowohl beim Patienten als auch beim Kostenträger.

- Die Ablehnungsrate durch Krankenkassen und die Versorgungslatenz sind bei den untersuchten ALS-Hilfsmitteln hoch. Beide Aspekte sollten in der Beratung aktiv thematisiert werden, um eine Demoralisierung der $\mathrm{Pa}$ tienten abzuwenden und die Option eines Widerspruchs gegenüber dem Kostenträger darzustellen.

- Ein Direktvergleich von fünf häufigen Kostenträgern der GKV zeigte große Unterschiede in der Ablehnungsrate von ALS-Hilfsmitteln. Hinweise auf eine „Zwei-Klassen-Medizin“ zugunsten der PKV ergaben sich nicht.

- Ablehnungs- und Latenzdaten zur Hilfsmittelversorgung einzelner Kostenträger, die aus digital unterstützten Versorgungsnetzwerken entstehen, können in der Zukunft ein Entscheidungskriterium für Versicherte sein, einen Wechsel innerhalb der GKV vorzunehmen.

Porrespondenzadresse
Ambulanz Tür ALS und andere
Motoneuronerkrankungen,
Neurologische Poliklinik,
CharitéCentrum für
Neurologie, Neurochirurgie
und Psychiatrie, Charité -
Universitätsmedizin Berlin,
Campus Virchow-Klinikum
Augustenburger Platz 1,
13353 Berlin
thomas.meyer@charite.de

Danksagung. Für die Unterstützung bei der Datenaufbereitung und statistischen Auswertung danken wir Doreen Wagner, PhD.

\section{Einhaltung ethischer Richtlinien}

Interessenkonflikt. T. Meyer und C. Münch sind Gründer des Internetportals Ambulanzpartner und Gesellschafter der Ambulanzpartner Soziotechnologie GmbH (APST), Berlin. Diese Studie wurde durch die folgenden Förderer unterstützt: Stiftung Stahlwerk Georgsmarienhütte, Initiative „Hilfe für ALS-kranke Menschen" und Angelika-von-Hagen-Fonds für ALS-Therapieforschung. Diese Untersuchung wurde durch die BMBF-Verbundvorhaben „MND-Net" (Förderkennzeichen: 01GM1103D), „,Mobile HybriCare" (01FG08003), ,'ServCare_ALS" (01FG09005) sowie der BMBF-geförderte EU-Projekt Joint Programme Neurodegenerative Disease Research (Förderkennzeichen 01ED1402 A) unterstützt (Pflichtmitteilung in englischer Sprache: This is an EU Joint Programme Neurodegenerative Disease Research project. The project is supported through the following funding organisations under the aegis of JPND [http://www.jpnd. eu]: Agency for Innovation by Science and Technology and Research Foundation, Flanders region; Bundesministerium für Bildung und Forschung, Germany; Economic and Social Research Council, United Kingdom; Health Research Board, Ireland; Ministry of Health and Ministry of Education, Universities and Research, Italy; The Netherlands Organization for Health Research and Development, The Netherlands [national regional organisations who are funding project, by country, in alphabetical order]). Die Ambulanzpartner Soziotechnologie $\mathrm{GmbH}$ erhielt eine Förderung durch die Technologie Stiftung Berlin (TSB) sowie das BMBF-Verbundprojekt "Innovationsdramaturgie nach dem Heldenprinzip" (Förderkennzeichen 01FH09159).

A. Funke, T. Grehl, J. Großkreutz, B. Walter, D. Kettemann, C. Karnapp, N. Gajewski, R. Meyer, A. Maier, K.M. Gruhn, T. Prel, K. Kollewe, S. Abdulla, X. Kobeleva, S. Körner und S. Petri geben an, dass kein Interessenkonfikt besteht.

Dieser Beitrag beinhaltet keine Studien an Menschen und Tieren.

\section{Open Access}

This article is distributed under the terms of the Creative Commons Attribution License which permits any use, distribution, and reproduction in any medium, provided the original author(s) and the source are credited.

\section{Literatur}

1. Ng L, Khan F, Mathers S (2009) Multidisciplinary care for adults with amyotrophic lateral sclerosis or motor neuron disease. Cochrane Database Syst Rev 4:CD007425

2. Meyer T, Großkreutz J, Münch C et al (2013) Ambulanzpartner - multiprofessionelles und internetunterstütztes Versorgungsmanagement bei der ALS. Klin Neurophysiol 44:159-166

3. Meyer T, Bienzeisler B, Klingner S (2014) Perspektiven einer informationstechnologischen Unterstützung personenbezogener Dienstleistungen. In: Bieber D, Geiger M (Hrsg) Personenbezogene Dienstleistungen im Kontext komplexer Wertschöpfung: Anwendungsfeld „Seltene Krankheiten". Springer Fachmedien Wiesbaden, S 134-149 
4. Swash M, Desai J (2000) Motor neuron disease: classification and nomenclature. Amyotroph Lateral Scler Other Motor Neuron Disord 1:105-112

5. Meyer T, Münch C, Landeghem FK van (2007) Progressive Muskelatrophie - eine unterdiagnostizierte Variante der Amyotrophen Lateralsklerose. Nervenarzt 78:1383-1388

6. Strong MJ, Gordon PH (2005) Primary lateral sclerosis, hereditary spastic paraplegia and amyotrophic lateral sclerosis: discrete entities or spectrum. Amyotroph Lateral Scler Other Motor Neuron Disord 6:8-16

7. Teijlingen ER van, Friend E, Kamal AD (2001) Service use and needs of people with motor neuron disease and their carers in Scotland. Health Soc Care Community 9:397-403

8. Bromberg MB, Brownell AA, Forshew DA, Swenson $M$ (2010) A timeline for predicting durable medical equipment needs and interventions for amyotrophic lateral sclerosis patients. Amyotroph Lateral Scler 11:110-115

9. Sanjak M, Bravver E, Bockenek WL et al (2010) Supported treadmill ambulation for amyotrophic lateral sclerosis: a pilot study. Arch Phys Med Rehabil 91:1920-1929

10. Brownlee A, Palovcak M (2007) The role of augmentative communication devices in the medical management of ALS. NeuroRehabilitation 22: 445-450

11. Heil- und Hilfsmittelreport der BARMER GEK https://presse.barmer-gek.de/barmer/web/Portale/Presseportal/Subportal/Infothek/Studienund-Reports/Heil-und-Hilfsmittelreport/Heil-undHilfsmittelreport-2014/Heil-und-Hilfsmittelreport-2014.html. Zugegriffen: 22. Apr. 2015

12. Kennzahlen durch den GKV-Spitzenverband. http://www.gkv-spitzenverband.de/me$\mathrm{dia} /$ grafiken/gkv_kennzahlen/kennzahlen_ gkv_2014_q2/GKV_Kennzahlen_Booklet_Q22014_300dpi_2014-09-16.pdf. Zugegriffen: 22. Apr. 2015

13. O'Brien MR, Whitehead B, Murphy PN et al (2012) Social services homecare for people with motor neuron disease/amyotrophic lateral sclerosis: why are such services used or refused? Palliat Med 26:123-131

14. Henschke C (2012) Provision and financing of assistive technology devices in Germany: a bureaucratic odyssey? The case of amyotrophic lateral sclerosis and Duchenne muscular dystrophy. Health Policy 105:176-184

15. Creemers H, Beelen A, Grupstra H et al (2014) The provision of assistive devices and home adaptations to patients with ALS in the Netherlands: patients' perspectives. Amyotroph Lateral Scler Frontotemporal Degener 15:420-425

16. Deutsche Vereinigung für Rehabilitation (2007) Für eine optimierte Versorgung mit Hilfsmitteln. Eine Expertise der Deutschen Vereinigung für Rehabilitation zu aktuellen Problemen bei der Versorgung mit Hilfsmitteln. Rehabilitation (Stuttg) 46:175186

\section{Neurologie für Afrika: Projekt zur Bekämpfung der Zystizer- kose}

Ein Projekt, das die Klinik für Neurologie und das Institut für Medizinische Mikrobiologie, Immunologie und Hygiene des Klinikums rechts der Isar der TU München gemeinsam mit afrikanischen Partnereinrichtungen durchführen, wird vom Bundesministerium für Bildung und Forschung (BMBF) mit rund sieben Millionen Euro gefördert. ."CYSTINETAfrica" beschäftigt sich mit der Bekämpfung der Zystizerkose in Tansania, Mosambik und Sambia. Zystizerkose ist eine infektiöse neurologische Erkrankung, die durch parasitäre Bandwürmer ausgelöst wird.

Das Ministerium fördert im Rahmen der Initiative „Forschungsnetzwerke für Gesundheitsinnovationen in Subsahara-Afrika“ insgesamt fünf afrikanisch-deutsche Forschungsnetzwerke, die aus über 70 Anträgen ausgewählt wurden. Das geplante Projekt besticht durch seine ausgeprägte Interdisziplinarität und eine internationale Ausrichtung.

Die unterschiedlichen Partner tragen verschiedene Bausteine rund um die Erforschung und Behandlung der Zystizerkose zusammen und erarbeiten gemeinsam neue Behandlungskonzepte unter Berücksichtigung des Erkrankungsverlaufs. Dabei soll die lokale Infrastruktur erweitert bzw. erneuert werden, Patienten eine adäquate Therapie zugänglich gemacht und die Ausbildung oder Promotion junger Akademiker gefördert werden. Die Wissenschaftler erhoffen sich zum einen, immunologisch-getriebene Pathomechanismen von parasitären Erkrankungen, die in erster Linie in Entwicklungsländern auftreten, in vivo beim Menschen als auch beim Tier aufzuklären. Aus den so gewonnenen Erkenntnissen könnten dann neue immunologische Modelle erstellt werden, um neurologisch relevante Fragen mittels der Grundlagenforschung effizienter anzugehen. Darüber hinaus sollen Grundlagen für eine effektive Behandlung der Patienten geschaffen werden.

\section{Fraunhofer IZI koordiniert EU- Projekt zu neurodegenerativen Erkrankungen}

Im Juni 2015 startete ein europäisches Verbundprojekt zur Aufklärung von molekularen Gemeinsamkeiten bei der Entstehung neurodegenerativer Erkrankungen. Fünf Partnereinrichtungen aus Halle, Leipzig, Erlangen, Paris und Oslo haben sich dabei zum Ziel gesetzt, basierend auf neuen Erkenntnissen Strategien für Therapien zu erarbeiten. Das Projekt mit einer Laufzeit von drei Jahren wird durch die Europäische Union mit insgesamt 1,7 Millionen Euro gefördert.

Neurodegenerative Erkrankungen ist gemeinsam, das sich Ihre Pathologie auf der Aggregation und Ansammlung fehlgefalteter Proteine im Gehirn begründet. Dadurch kommt es zu Gewebe- und Funktionsverlust und folglich zu schweren neurologischen Störungen, die bei Fortschreiten der Krankheit zum Tode führen. Bisher wurden die Mechanismen der Entstehung dieser Krankheiten vorwiegend unabhängig voneinander untersucht. Neuere Untersuchungen legen jedoch Gemeinsamkeiten in den molekularen Entwicklungsprozessen der Krankheiten nahe. Im Mittelpunkt steht dabei die Hypothese, dass fehlgefaltete Peptide, die gehäuft bei einer Krankheit entstehen, auch eine Ablagerung anderer Eiweiße induzieren. Das heißt, ein Peptid löst degenerative Prozesse aus, welche sich durch Ko-Aggregation der Peptide verstärken. Zu den wichtigsten Zielproteinen, die untersucht werden sollen, gehören zum Beispiel Abeta, alpha-Synuclein und Huntingtin.

Dieses Projekt wird gefördert durch das “EU Joint Programme - Neurodegenerative Disease Research (JPND)", der größte globalen Forschungsinitiative, welches die Herausforderungen neurodegenerativer Erkrankungen adressiert.

Weitere Informationen: www.jpnd.eu

Quelle:www.izi.fraunhofer.de 